\title{
Coulomb scattering of quantum dipoles in QED.
}

\author{
B. Blok* \\ Department of Physics, Technion - Israel Institute of Technology, Haifa 32000, Israel
}

\begin{abstract}
We calculate the total scattering cross-section of a dynamical quantum electrically neutral dipole in QED of the infinitely heavy charge and of the infinitely heavy dipole in the leading order in $\alpha_{e m}$.
\end{abstract}

*E-mail: blok@physics.technion.ac.il 


\section{INTRODUCTION}

The study of the space-time evolution of quantum dipoles in QED and QCD attracted a lot of attention recently $[1-6,8,7]$.

In particular in a recent paper [7] we have showed that the evolution of the approximately symmetric quantum dipole can be described using the renormalized wave function. The purpose of the present paper is to use the formalism developed in ref. [7] to calculate the cross-section of a quantum dipole off the Coulomb center and off the other dipole.

Our main result is the use of the regularized dipole wave function of ref. [7] for the precise definition of the dynamical dipole time-dependent cross-section and it's calculation. We obtain

$$
\sigma(T)=g^{2}(S(T) / 2) \log (E / \delta)
$$

for the scattering of the approximately symmetric dynamical dipole with the center of mass energy E of the infinitely heavy Coulomb center, and

$$
d \sigma(T) / d t=g^{2} S(T) b^{2} / 2
$$

for the scattering of the dynamical dipole of the infinitely heavy dipole. Here $S(T)$ is the transverse area of the dynamical dipole at time $\mathrm{T}$ after its creation, and $\mathrm{b}$ is the transverse radius of the target dipole.

Our results are in agreement with the ones by D.Soper and J.Gunion [10,1] that the scattering cross-section off the dipole is proportional to $b^{2}$, and the well known statement that the dipole cross-section of the charged Coulomb center increases logarithmically with energy [11-13]. In the leading order our cross-section coincides with the one obtained in ref. [1] in a different way.

The paper is organized in the following way. In the second chapter we discuss the general formalism of calculations. In the third chapter we carry the calculations and our results are summarized in conclusion.

\section{GENERAL FORMALISM}

\section{A. Dipole way function.}

It was explained in ref. [7] that one can describe the dipole evolution by means of the $a, b \rightarrow 0$ limit of the dipole wave function

$$
\begin{aligned}
\Phi(\vec{r}, \vec{R}, t) & =(1 / \sqrt{2 E}) \sqrt{2 a / \pi} \exp (i \vec{Q} \vec{R}) \exp (i E t) \int d^{2} q_{t} \exp \left(-(a+i b)\left(\vec{q}_{t}-\vec{k}_{t}\right)^{2}\right. \\
& \left.+2(i t /(E)) q_{t}^{2}\right) \\
& \times \exp \left(i \overrightarrow{q_{t}} \vec{\rho}\right)
\end{aligned}
$$


(where we neglect the frozen evolution in z direction). In coordinate space one has

$$
\begin{aligned}
\Phi(r, R, t) & =(1 / \sqrt{2 E}) \exp (i \vec{Q} \vec{R}) \exp (i E t) \frac{\sqrt{2 a \pi}}{a+i(b+4 t / E)} \\
& \times \exp \left(-\left(\vec{\rho}-4 \vec{k}_{t} t / E\right)^{2} /\left((a+i(b+4 t / E))+i \vec{k}_{t}\left(\vec{\rho}-4 \vec{k}_{t} t / E\right)\right) .\right.
\end{aligned}
$$

For this dipole we have time dependent radius:

$$
\begin{gathered}
<\rho^{2}(t)>=a / 2+b^{2} / 2 a+8(b / a) \hbar t / E+8 t^{2} /\left(E^{2} a\right) \\
\rho^{i}(t)=4 k^{i} t / E .
\end{gathered}
$$

Our strategy will be to use these wave functions in order to calculate scattering amplitudes. We shall limit ourselves here with the most interesting case of quantum dipole $\left(\vec{p}_{t}=0\right)$.

\section{B. Scattering amplitude calculation.}

It was explained in ref. [9] ( see also the discussion relevant to the given case in ref. [7]) that in order to calculate the amplitude, one can use casual ordering of the old perturbation theory [14]. All other contributions are suppressed by the powers of energy.

\section{Time dependent cross-section.}

We are interested in the full cross-section that is proportional to

$$
\partial P(T) / \partial T
$$

Here $\mathrm{P}(\mathrm{T})$ is the probability of all possible transitions in time $\mathrm{T}$

$$
P(T)=\sum_{f} M_{i f} M_{i f}^{*}
$$

where $\mathrm{i}$ is our initial dipole configuration, and $f$ is a full system of intermediate states. Since we carry our calculations using free wave packets we can take as a full system the free particle wave functions:

$$
\left.\psi\left(\vec{r}_{1}, \vec{r}_{2}\right)=\frac{1}{\sqrt{\epsilon_{p_{1}} \epsilon_{p_{2}}}} \exp \left(i \vec{k}_{1} \vec{r}_{1}\right)+i \vec{p}_{2} \vec{r}_{2}\right)+i \epsilon_{p_{1}} t-i \epsilon_{p_{2}} t
$$

where $\epsilon_{p}=\sqrt{p^{2}+M^{2}}$. Then the summation over the intermediate states reduces to integration over $d^{3} p$. The time-dependent cross-section is defined as

$$
\sigma(T)=\partial P(T) / \partial T
$$

Here the wave functions of the dipole constituents must be normalized like $1 / \sqrt{V}$, corresponding to one particle in a unit volume. 


\section{CALCULATION OF THE SCATTERING CROSS-SECTION}

\section{A. Scattering of the infinitely heavy Coulomb center.}

In order to calculate the scattering cross-section we need to calculate the difference of transition amplitudes from the initial dipole to a $\vec{p}_{1} \vec{p}_{2}$ intermediate state, take a square and then integrate over $d^{2} p_{1} d^{3} p_{2}$. It is easy to see that the corresponding amplitude is just the difference

$$
\begin{aligned}
M & =\sqrt{2 a / \pi} \int d s_{0}^{T}\left(\operatorname { e x p } \left(\left(a(p-k)^{2}+2 i\left(2 k_{t} p_{t}+k_{t}^{2}\right) t / E\right.\right.\right. \\
& -\exp \left(-\left(a(p+k)^{2}+2 i\left(-2 k_{t} p_{t}+k_{t}^{2}\right) t / E\right)\right.
\end{aligned}
$$

Here $\vec{p}=\vec{p}_{1}-\vec{p}_{2}$ and $\vec{k}$ is a transferred momenta during the scattering. The first term in the latter expression corresponds to the Coulomb photon emission from a positive charged component of the dipole (a particle) while the second-from the negative charge one. The square of this expression is given by the difference $M_{1}-M_{2}$, corresponding to direct and cross product, i.e. in the first term we consider the product of amplitudes corresponding both to particle, or both to antiparticle, and the second term corresponds to the product when one of the amplitudes corresponds to particle and the other to antiparticle. It is easy to obtain

$$
\begin{aligned}
M_{1} & \left.\left.=\int^{d} t \int d t^{\prime} \int d^{2} p_{t} \exp \left(2 i\left(p_{t}+k\right)^{2}\left(t-t^{\prime}\right)-2 i p_{t}^{2}\left(t-t^{\prime}\right)\right) / E\right)+i k_{3}\left(t-t^{\prime}\right)\right) \\
& \times \exp \left(-(a+i b)\left(p_{t}-k\right)^{2}+\left(p_{t}+k\right)^{2}\right) \sqrt{2 a / \pi}
\end{aligned}
$$

The second diagram is given by

$$
\begin{aligned}
M_{2} & \left.=\int_{0}^{T} d t \int d t^{\prime} \int d^{2} p_{t} \exp \left(i\left(p_{t}+k_{t}\right)^{2}-p_{t}^{2}\right) t / E-i\left(\left(p_{t}-k_{t}\right)^{2}-p_{t}^{2}\right) t^{\prime} / E\right) \\
& \left.+i k_{3}\left(t-t^{\prime}\right)\right) \exp \left(-(a+i b)\left(p_{t}+k_{t}\right)^{2}-(a-i b)\left(p_{t}-k_{t}\right)^{2}\right)(2 a / \pi) /\left(k^{2}+\delta^{2}\right)^{2} d 3 k
\end{aligned}
$$

We can carry the integration over the transverse momentum $p_{t}$. We obtain:

$$
\begin{gathered}
M_{1}=\int d t d t^{\prime} \exp \left(2 a-2 k_{t}^{2} s^{2} /\left(E^{2} a\right)+i k_{t}^{2} s / E+i k_{3} s /\left(k^{2}+\delta^{2}\right)^{2}+h . c .\right. \\
\left.M_{2}=\int d t \int d t^{\prime} \exp \left(-2\left(4\left(t+t^{\prime}\right) / E+b\right)\right)^{2} k_{t}^{2} /(a)+i k_{t}^{2} / E+i k_{3} s\right)+ \text { h.c. }
\end{gathered}
$$


We now differentiate $M_{1}$ and note that if $T \ll \sqrt{E^{2} a} / k_{t}$, we can neglect the real term in the argument of the exponent in eq. 3.5. The integration over $\mathrm{s}$ then gives the result that is peaked near $0=k_{3}+k_{t}^{2} / E$. We can approximately substitute the result of this integration by a $\pi \delta\left(k_{3}+k_{t}^{2} / E\right.$, and then carry the integration over $k_{3}$. The net result since $k_{t} \ll E$, is that we can put $k_{3}=0$. Consider now the eq. 3.6. There we can put after differentiation approximately $u=T+t^{\prime} \sim 2 T$, while the integration over s and $k_{3}$ as before gives us $k_{3}=0$. Thus we obtain

$$
\sigma(T) \equiv \partial\left(M_{1}-M_{2}\right) \partial T=2 \pi \int d^{2} k_{t}\left(2 a-\exp \left(2(4 i T / E+i b)^{2} k_{t}^{2}\right) / a\right) /\left(k_{t}^{2}+\delta^{2}\right)^{2}
$$

for times much smaller than the coherence length we can expand the exponents in eqs. (3.6-10). and obtain

$$
\sigma(T)=4 \pi \int d^{2} k_{t}\left(a^{2}+((4 T / E+b))^{2} k_{t}^{2} / a\right) /\left(k_{t}^{2}+\delta^{2}\right)^{2}
$$

\section{B. Renormalization.}

The latter calculation is however not an end of the story. It was explained in ref. [7] that we must now go to the limit $a, b \rightarrow 0,8 a / b \rightarrow D, E^{2} a \rightarrow v_{t}^{2}$ to keep gauge invariance of an amplitude. Then one immediately obtains

$$
\sigma(T)=\pi \int d^{2} k_{t} / k_{t}^{2} \times\left(D T / E+v_{t}^{2} T^{2}\right)
$$

Here $D$ is the diffusion coefficient ( $D=2$ for the longitudinal vector current $[1,15]$ ). In other words the total cross-section is just

$$
\sigma(T)=S(T) \int d k_{t} / k_{t}
$$

integrating the latter equation with logarithmic accuracy we obtain using $k_{t}=2 E \sin (\theta / 2)$

$$
P(T)=S(T) \log (E / \delta)
$$

Here $S(T)$ is the dipole transverse area at time T, calculated in ref. [1,7]:

$$
S(T)=D T / E+v_{t}^{2}
$$

It is now clear what is the relevant time scale:

$$
T \ll 1 /\left(v_{t} k_{t}\right)=E /\left(p_{t} k_{t}\right)
$$

Here $v_{t}=p_{t} / E$ is a renormalized characteristic transverse momentum of the dipole. 


\section{Dipole-dipole scattering.}

In the previous section we carried the calculation for the scattering on the infinitely heavy Coulomb center. Exactly the same calculation can be made for the dipole-dipole scattering on the infinitely heavy dipole with the transverse scale b. It is straightforward to see that the dipole contributes the multiplier

$$
\left|1-\exp \left(i \overrightarrow{k_{t}} \vec{b}\right)\right|^{2}=4 \sin \left(\overrightarrow{k_{t}} \vec{b} / 2\right)^{2}
$$

For small $b$ we can expand the latter sinus in Taylor series and obtain

$$
\sigma(T)=\sigma(T) b^{2} \int d k_{t} k_{t}
$$

We can move from integration over $k_{t}=2 E \sin (\theta / 2)$ to integration over the invariant kinematic variable $t=k_{t}^{2}$. Then

$$
\frac{d P(T)}{d t}=\sigma(T) b^{2} / 2
$$

We see that the differential cross-section of dipole-dipole scattering does not increase with energy, while the full cross-section must be determined through the integration over the allowed kinematic range of $t$. This is in contrast to the scattering on the infinitely heavy coulomb center, where the full cross-section increased logarithmically with energy.

Our result 3.16 in the leading order coincides with the one by Farrar et al [1] (see also ref. [15]) obtained in a different way.

\section{CONCLUSION.}

In this paper we have shown that using the dipole wave functions defined in ref. [7] and old perturbation theory [14], it is possible to calculate the scattering cross-section of the dipole of the infinitely heavy dipole and of the coulomb center.

Our main result is the precise definition of the dynamical dipole time-dependent crosssection as a time-dependent matrix element between the wave functions of ref. [7] and it's calculation:

$$
\sigma(T)=g^{2} S(T) \log (E / \delta)
$$

for the scattering of the dynamical dipole of the infinitely heavy Coulomb center, and

$$
d \sigma(T) / d t=g^{2} S(T) b^{2} / 2
$$

The result coincides in the leading order with the one due to ref. [1] that was obtained in a different way. The dependence on the target dipole size for the scattering of the heavy dipole and on the energy coincide with the known general results due to [10-13].

Our results can be easily extended to the scattering of the dynamical dipole in the strong field. The only difference is that instead of a full system of the plain waves used here one must use the the full system of the wave functions in this external field. 


\section{ACKNOWLEDGMENTS}

The author thanks prof. L. Frankfurt for reading a manuscript and numerous useful discussions. 


\section{REFERENCES}

[1] G. R. Farrar , H. Liu, L. Frankfurt and M. Strikman, Phys. Rev. Lett.,61 (1988) 686; $62(1989) 387$.

[2] L. Frankfurt and M. Strikman, Progress in Part. Nucl. Phys., 27 (1991) 135.

[3] L. Frankfurt, J. Miller, M. Strikman, Annual review on Particle and Nuclear Physics, 44 (1994) 501.

[4] L. Frankfurt and M. Strikman, Phys. Rept., 160 (1988) 235.

[5] A.H. Mueller, Nucl. Phys., B415 (1994) 373; B437 (1995) 107.

[6] V.N. Gribov, Nucl. Phys.,B206,103 (1982).

[7] B.Blok Space-Time evolution of Quantum dipoles in Quantum electrodynamics, preprint hep-ph/0404117, submitted to Phys. Rev. D.

[8] B. Blok, Phys. Rev., D69 (2004) 022003.

[9] V. Gribov, in proceedings of the 8th LNPI Winter scool in physics, pp.5 (1973), reprinted in V. Gribov, Gauge theories and quark confinement, p. 3, Moscow, Phassis, 2002.

[10] A. Gunion and D. Soper, Phys. Rev., D15 (1977) 2617.

[11] H. Bethe and W. Heitler, Proc. Roy. Soc. 146 (1934) 83.

[12] V. Berestetskii, E. Lifshits, L. Pitaevskii, Quantum Electrodynamics, Oxford, Pergamon Press, 1982.

[13] A.I. Akhieser and V. Berestetskii, Quantum Electrodynamics, Interscience, New York (1965).

[14] B. Heitler, The Quantum Theory of Radiation, Oxford, 1954; W.T. Grandy, Introduction to electrodynamics and radiation, Academic Press, 1970, New York and London.

[15] Yu. L. Dokshitser et al,Basics of Perturbative QCD. Edition Frontieres, Gif-Sur-Yvette Cedex, France, 1991. 